\title{
Erratum
}

\section{Analysis of a retraction mechanism for imbedded polymeric fibers}

\section{Rheologica Acta 28(3), 223-232 (1989)}

A. Cohen and C. J. Carriere

The Dow Chemical Company, Midland, Michigan, USA

1. The differential equation for the normalized radius $r$ at the bottom of page 228 should read as

$$
\frac{\left(4-r^{3}\right)^{2}}{\left(1-r^{3}\right) r^{3}} \frac{d r}{d t}=\frac{1}{\lambda} .
$$

2. The correct value for the hydrodynamic factor $\chi$ should be $3.5 / 6 \pi$ and not just 3.5 as stated in pp. 231 and 232 .

Authors' address:

Dr. Aric Cohen, Dr. Craig J. Carriere The Dow Chemical Company Central Research, 1202 Building Midland MI 49624, USA 